\title{
Theory and computation of the matrix elements of the full interaction of the electromagnetic field with an atomic state: Application to the Rydberg and the continuous spectrum
}

\author{
Yannis Komninos, ${ }^{1}$ Theodoros Mercouris, ${ }^{1}$ and Cleanthes A. Nicolaides ${ }^{1,2}$ \\ ${ }^{1}$ Theoretical and Physical Chemistry Institute, National Hellenic Research Foundation, 48 Vasileos Constantinou Avenue, \\ Athens 116 35, Greece \\ ${ }^{2}$ Physics Department, National Technical University, Athens, Greece
}

(Received 30 October 2001; published 5 April 2002)

\begin{abstract}
We develop practical formulas for the calculation of the matrix elements of the interaction of the electromagnetic field with an atomic state, beyond the long-wavelength approximation. The atom-plus-field Hamiltonian is chosen to have the multipolar form, containing the electric, paramagnetic, and diamagnetic operators. The final workable expressions include the interactions to all orders and are derived by first expanding the fields in partial waves. The electric-field operator reaches a constant value as the radial variable becomes large, contrary to the result of the electric-dipole approximation (EDA) where the value of the corresponding operator increases indefinitely. Applications are given for Rydberg states of hydrogen up to $n=50$ and for free-free transitions in a Coulomb potential. Such matrix elements are relevant to a number of real and virtual processes occurring during laser-atom interactions. The computation is done numerically, using a combination of analytic with numerical techniques. By comparing the results of the EDA with those of the exact treatment, it is shown that the former is inadequate in such cases. This finding has repercussions on the theory and understanding of the physics of quantum systems in high-lying Rydberg levels and wave packets or in scattering states.
\end{abstract}

DOI: 10.1103/PhysRevA.65.043412

PACS number(s): 32.80.Rm, 33.80.Rv

\section{THE LONG-WAVELENGTH APPROXIMATION AND EXTENDED WAVE FUNCTIONS}

As is well known, theoretical atomic and molecular spectroscopy of a plethora of phenomena caused by the linear and nonlinear interaction of atomic (molecular) states with the electromagnetic field has been developed over the decades using the lowest-order terms of the angularmomentum- and parity-dependent multipole expansion of the vector potential [1-3]. The fundamental justification of this framework is the validity of the long-wavelength approximation (LWA) and the concomitant physical and computational relevance of matrix elements of the electric-dipole $(E 1)$, electric-quadrupole (E2), etc., or of the magnetic-dipole (M1), magnetic-quadrupole (M2), etc., operators. These matrix elements enter into various theoretical expressions of perturbative or nonperturbative treatments, either by themselves or in combination (e.g., interference of $E 2$ with $M 1$ amplitudes), for the calculation of real or virtual processes.

The LWA is based on the condition that $2 \pi r / \lambda$ is much smaller than unity. $\lambda$ is the wavelength of radiation and $r$ signifies the "atomic dimensions" of the two states (real or virtual) involved in the transition matrix element. Under this condition, the low-order expansion of the vector potential $\vec{A}(\vec{r})$ taken in the transverse gauge is valid:

$$
\begin{aligned}
& \vec{A}(\vec{r})=\vec{\epsilon}_{k \mu} e^{i \vec{k} \cdot \vec{r}}, \quad \vec{\epsilon}_{k \mu} \cdot \vec{k}=0, \quad \mu=1,2, \\
& \vec{A}(\vec{r})=\vec{\epsilon}_{k \mu}[1+\vec{k} \cdot \vec{r}+\cdots] \quad \text { (LWA). }
\end{aligned}
$$

$\vec{\epsilon}_{k \mu}$ is the polarization vector, $\mu$ numbers the two possible transverse polarizations of the field, and $\vec{k}$ is the photon wave vector.
The application of the LWA in quantum mechanics is extensive and pervades many fields, especially in the form of the electric-dipole approximation (EDA), whereby only the first term of Eq. (2) is kept. Its overall success in conventional spectroscopy of states of small spatial extent is indisputable. Furthermore, the simple forms that its operators acquire in terms of their radial dependence, such as the length or the velocity operators of the EDA, have made the computation of matrix elements between polyelectronic wave functions rather straightforward.

However, it appears that there are domains of currently applied laser spectroscopy where the LWA and its most conspicuous consequence, the EDA, cannot be justified a priori. Such domains are defined by spectroscopies probing Rydberg states, and by strong-field laser spectroscopy, using a laser (and its harmonics) of frequencies ranging from the infrared to the uv and vuv. In these cases, one may have to calculate quantities involving the following types of matrix elements: Rydberg-Rydberg state, Rydberg-scattering state, and scattering-scattering state. The dimensions of such states are either huge (say, Rydberg levels of hydrogen with $n$ $=50-100$ ), or unlimited (energy-normalized scattering states), and have nothing to do with what is usually termed the "atomic dimensions." Therefore, their extent becomes commensurate with that of the wavelengths of the commonly used radiation sources and hence, when considering interaction matrix elements involving such functions, the condition of the LWA cannot be satisfied. Therefore, results on fieldinduced processes that involve such extended states and are based on the EDA cannot be justified a priori. This comment was made in a relatively recent publication [4] on the theory and computation of free-free transition matrix elements within the EDA. At the same time, we also conjectured that, given the apparent agreement between computations in the EDA and experimental phenomenology of processes involv- 
ing free-free transitions, the interpretation of the physics might be that the major part of the interaction occurs between the field and an electron wave packet very close to the nucleus.

Questions and ambiguities of this nature cannot be answered definitively without calculation of atom-field coupling matrix elements beyond the EDA and without computation of the corresponding, physically relevant wave functions, transition probabilities, and rates. The present work is a contribution toward this goal. It was motivated by recent publications [5-9] where, starting with Corless and Stroud [5], the aim of the calculations and of the discussions is to obtain useful information about the formation of angular wave packets in high- $n$ hydrogenic states and about photoionization of high- $n$ states, using wave functions from only one $n$ manifold or from more such manifolds. In all cases, the analysis and calculations were done in the EDA. The work of [5-8] used the length form of the interaction and that of [9] used the velocity form.

The only quantitative clue as to what happens when one goes beyond the EDA in order to compute the matrix elements between such high- $n$ Rydberg states has been provided thus far by the recent work of Madsen, Hansen, and Nilsen [10], whose results confirm the conjecture that in such cases the EDA is inadequate. Specifically, Madsen et al. [10] compared the values of the matrix elements of the velocity operator of the EDA, $\vec{A}(0) \cdot \vec{p}$, with those of $\vec{A}(\vec{r}) \cdot \vec{p}$, present in the "minimal-coupling" Hamiltonian [1-3], for hydrogen levels with $n$ up to 30. The quadratic term of the interaction, $A^{2}(\vec{r})$, whose significance increases with increasing field strength, was not considered. Their results show that, for matrix elements within Rydberg manifolds with $n$ larger than about 10-15 (Fig. 1 of [10]), the EDA is not reliable. Specifically, Madsen et al. [10] stated that when $n=30$ the EDA "breaks down in connection with laser excitation of angular wave packets."

If one aims at developing a practical formalism for going beyond the EDA for treating problems of hydrogen Rydberg wave-packet dynamics such as the ones proposed and examined in [5-9], it is necessary to choose a consistent and pertinent form for the Rydberg state-field interaction. For example, consider the single $n$ manifold model employed by Corless and Stroud [5] for determining the probability of forming high-angular-momentum wave packets. They used the length form of the EDA, for which the intrashell dipole matrix elements are huge. (They are given by $-\frac{3}{2} n \sqrt{n^{2}-l^{2}}$, regardless of the coordinate system-spherical or parabolic. Obviously, they increase rapidly with $n$; see also Sec. IV.) On the contrary, if the EDA is chosen in the velocity form, these intrashell dipole matrix elements are zero. This can easily be seen from the relation $\langle\vec{p}\rangle=\left(E_{i}-E_{j}\right)\langle\vec{r}\rangle$, where, for hydrogen, $E_{i}=E_{j}$ within the same $n$. Given the length values, Corless and Stroud [5] argued that the attenuation of the results of this zero-order choice by the coupling of the $n$ th-shell levels with those of the other shells and with the continuum would not affect the conclusions significantly. This assumption led to additional calculations and to counterarguments [6-9], and the problem is basically open.

On the other hand, this model, namely, the neglect of the intershell electric-dipole matrix elements, is meaningless in the velocity form of the EDA. This is because now the opposite is true, namely, the intrashell matrix elements are zero while the intershell ones are important. Since the latter are unlimited in number, the time-dependent calculation appears to acquire large dimensions for which no computational experience has been published yet for large $n$. This feature characterizes the $\vec{A} \cdot \vec{p}$ operator as well. Of course, as regards the final result of a calculation that solves the time-dependent Schrödinger equation (TDSE) with all the states (including the continuum) coupled, the EDA forms of the length and velocity operators ought to be equivalent. In practice, the function spaces are truncated and the interesting question is how to obtain quantitatively the correct physics from the solution of the TDSE in an economic way. Here, we point out that questions about the significance of intermediate states in problems where either the length or the velocity form is used were also dealt with by Lamb, Schlicher, and Scully [11], who studied the two-photon $1 s-2 s$ transition in hydrogen. For example, they noted that "the contributions of the individual intermediate states are very different" when the two forms are used (Appendix B of [11]).

Because of the above, we decided to treat the computational problem of "EDA vs beyond EDA" in a rigorous manner to all orders, via the use of a multipolar Hamiltonian [2,3,11-13]; see Sec. III. In what follows, we present our approach, formalism, and numerical results for the calculation of matrix elements beyond the LWA and the EDA. The demonstrative applications concern the electric-dipoleallowed $s \rightarrow p$ and the dipole-forbidden $s \rightarrow d$ matrix elements in the Rydberg spectrum and in the continuous spectrum of hydrogen.

\section{PRESENT WORK: CHOICE OF THE MULTIPOLAR HAMILTONIAN}

An approximate approach for going beyond the EDA would be to employ higher-order terms in the expansion (2) for weak as well as for strong fields. This may be easily stated but is not easily doable, especially for high-lying states where there are energy degeneracies and near degeneracies. Furthermore, especially for strong fields, one would have to prove convergence of the series for each case of interest.

In the present paper we give a practical formalism for the expressions and the calculation of the nonrelativistic matrix element of the full interaction between an atomic state and the electromagnetic field, which does not employ any type of multipole expansion and which is generally applicable, including to the unexplored cases of high-lying states. Both the Rydberg orbitals $\left|R^{n}\right\rangle$ and the energy-normalized scattering orbitals $|E\rangle$ are considered to be in numerical form. As in our previous work on atomic structure and dynamics, we develop the formalism and methodology in terms of numerical orbitals, rather than analytic hydrogenic ones, so as to treat the general $N$-electron problem, where $\left|R^{n}\right\rangle$ or $|E\rangle$ is calculated from state-specific $(N-1)$-electron potentials.

The determination of such full-interaction matrix elements also entails the deduction of their analytic properties. 
For example, if we need to compute the $\langle E|$ operator $\left|E^{\prime}\right\rangle$, for $E=E^{\prime}$ or for $E \neq E^{\prime}$, we need to recognize and deal with the possible existence of singularities, as in the case of the EDA or of the full minimal-coupling interaction ([4] and references therein).

The form of the Hamiltonian describing the atom-field interaction that was chosen for this work is the multipolar one $[2,3,11-13]$. This Hamiltonian is equivalent to the conventional minimal-coupling atom-field Hamiltonian via unitary or gauge transformations. In the limit of the EDA, it reduces to the length form of the electric-dipole interaction, i.e., $\vec{E}(0) \cdot \vec{r}$, where $\vec{E}(0)$ is the field at $r=0$.

The multipolar Hamiltonian has been studied in the past in the form of an infinite sum, whose terms represent electric or magnetic multipoles, with the purpose of keeping and analyzing the first few of them. The elegant presentation of Loudon [2] leads to a compact form in terms of the integral of a quantity $0<\lambda<1$, multiplying the position vector [Eq. (4) below]. The Taylor expansion of the position-dependent fields reproduces the terms of the infinite multipolar sum. The $\lambda$ integral can be evaluated by a combination of analytic and numerical methods-see Eq. (6) and below. The overall result is a computationally practical form of the multipolar Hamiltonian.

Having obtained the appropriate expressions, we computed numerically the matrix elements involving $\left|R^{n}\right\rangle$ as well as $|E\rangle$ of hydrogenic states, and compared them with the results obtained from the application of the EDA in the length form. There are strong discrepancies, suggesting that the theory and computation of related phenomena and observables ought to take this fact into account, and that published conclusions on such phenomena, involving the coupling of the electromagnetic field with the continuous and the high-Rydberg spectra, need reexamination. Applications to the dynamics of highly excited Rydberg wave packets will be given in a separate publication.

\section{PRACTICAL EXPRESSIONS FOR THE MATRIX ELEMENTS OF THE FULL INTERACTION OF THE MULTIPOLAR HAMILTONIAN}

The interaction part of the conventional, minimalcoupling Hamiltonian is given by

$$
H_{I}=\frac{e}{m c} \sum_{j} \vec{A}\left(\vec{r}_{j}\right) \cdot \vec{p}_{j}+\frac{e^{2}}{2 m c^{2}} \sum_{j} \vec{A}^{2}\left(\vec{r}_{j}\right) .
$$

As we discussed in Sec. I, the use of Eq. (3) in calculations of certain interesting problems of wave-packet dynamics need not provide an optimal treatment. In addition, one must consider the fact that, when using Eq. (3) for solving the TDSE, the expansion coefficients of a basis set of the unperturbed Hamiltonian are gauge dependent and do not correspond directly to time-dependent amplitudes $[11,15]$. In addition, as Lamb, Schlicher, and Scully [11] pointed out, the turning on and off of the interaction must be assumed to occur adiabatically. Therefore, after some analysis, we opted for the investigation of the multipolar Hamiltonian that re- duces directly to the length form of the EDA, and which is obtained from Eq. (3) by the application of the unitary transformation $[2,3]$

$$
U=\exp \left\{\frac{i}{h c} \sum_{j} \int_{0}^{1} \vec{r}_{j} \cdot \vec{A}\left(\lambda \vec{r}_{j}\right) d \lambda\right\} .
$$

The multipolar Hamiltonian is given by $[2,3,12-14]$

$$
\begin{aligned}
H_{I}= & e \sum_{j} \int_{0}^{1} \vec{r}_{j} \cdot \vec{E}_{T}\left(\lambda \vec{r}_{j}\right) d \lambda-\frac{e}{m c} \sum_{j} \int_{0}^{1} \lambda \vec{p}_{j} \cdot \vec{r}_{j} \\
& \times \vec{B}_{j}\left(\lambda \vec{r}_{j}\right) d \lambda+\frac{e^{2}}{2 m c^{2}} \\
& \times\left\{\sum_{j} \int_{0}^{1} \lambda \vec{r}_{j} \times \vec{B}_{j}\left(\lambda \vec{r}_{j}\right) d \lambda\right\}^{2}
\end{aligned}
$$

and involves the electric and magnetic fields rather than the vector potential. The $\lambda$ integration permits the writing of expressions involving infinite expansions in a compact form.

The three terms above are the electric, paramagnetic, and diamagnetic operators. In what follows, we show how to reduce Eq. (5) to workable expressions for the computation of matrix elements.

The previous treatments of the above expression $[2,3,12-$ 14] involve the Taylor expansion about the origin of the fields, described by plane waves, followed by the evaluation of the $\lambda$ integrals. Keeping the first term of the electric-field expansion, one obtains the length form of the EDA. Here we shall follow a different route. We will use the partial-wave expansion (A4) of the Appendix of the plane waves describing the fields, since this expansion involves the spherical harmonics, which are also used to describe the angular part of the atomic orbitals. In this way, the integration over angles is evaluated analytically in terms of the $3-j$ symbols and the infinite expansion is truncated by the triangular inequalities $\left|l_{i}-l_{f}\right| \leqslant l \leqslant l_{i}+l_{f}$ between the angular momenta included in the 3-j symbols, where $l_{i}$ and $l_{f}$ are the initial and final angular momenta, respectively. Because of another property, $l$ $+l_{i}+l_{f}$ must be even. Consequently, the index $l$ in the expansion increases in steps of 2 and the summation is either a real or a purely imaginary number. For example, in the case of the dipole-allowed transitions $l_{f}=l_{i} \pm 1$ and therefore $l$ $=1,3, \ldots, 2 l_{i} \pm 1$.

In the next step, the $\lambda$ integrals are reexpressed as integrals over the radial variable,

$$
\int_{0}^{1} j_{l}(\lambda k r) \lambda^{n} d \lambda=\frac{1}{r^{n+1}} \int_{0}^{r} j_{l}\left(k r^{\prime}\right) r^{\prime n} d r^{\prime}
$$

where the Bessel functions result from the partial-wave expansion of the plane waves. For reasons of simplicity the $z$ axis is chosen in the direction of the wave vector $\vec{k}$. For the polarization of the fields $E$ and $B$ we choose the $x$ and $y$ axes, respectively; see formulas (A1)-(A3) of the Appendix. Use is also made of the simplifying formulas (A5) and (A6).

The result of these choices is that the operators of the atom-field interaction are put in the form 


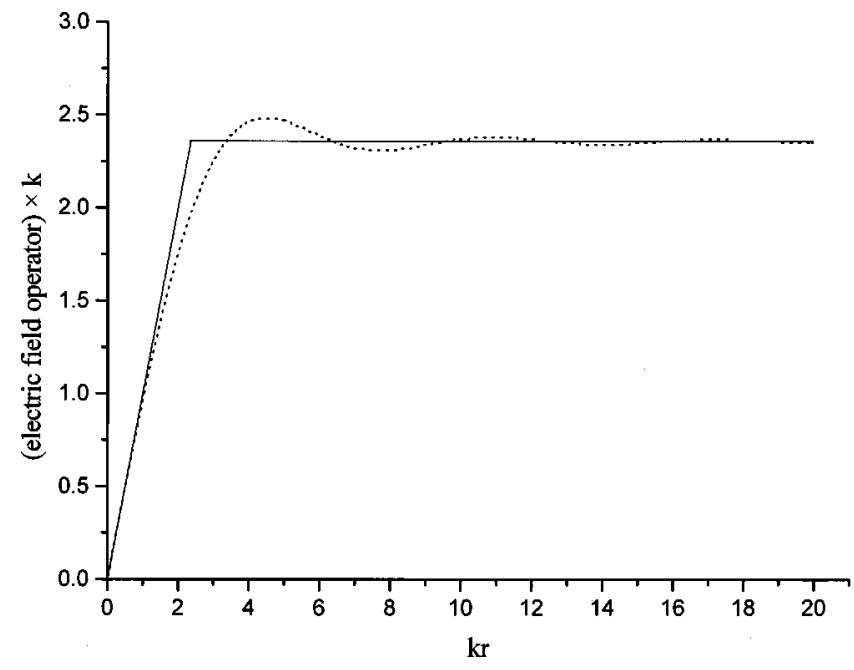

FIG. 1. Plot of the full electric operator $3 F_{1}$ Eq. (8) (dotted line) versus the model operator Eq. (12) (full line), both multiplied by the photon wave number.

$$
O_{X}=X(t) \sum_{l=l_{\min }}^{\infty} i^{l+l_{\min }}(2 l+1) F_{l}(r) \Theta_{l}(\theta, \phi)+\text { c.c. }
$$

\section{A. Electric-field operator}

Specifically, for the electric-field operator $\left[l_{\min }=1, X(t)\right.$ $\left.=E_{0}(t) e^{-i \omega t}\right]$ the application of Eq. (A5) gives

$$
F_{l}(r)=\frac{1}{k} \int_{0}^{r} \frac{1}{r^{\prime}} j_{l}\left(k r^{\prime}\right) d r^{\prime}
$$

and

$$
\Theta_{l}(\theta, \phi)=-\sqrt{\pi} \sqrt{l(l+1) /(2 l+1)}\left(Y_{l}^{1}-Y_{l}^{-1}\right),
$$

causing transitions with $\Delta m= \pm 1$. For values of $k \rightarrow 0$, only the first term survives.

A useful insight is gained by examining the large- $r$ behavior of $F_{l}$. We write

$$
F_{l}(r) \rightarrow \frac{1}{k} c_{l}-\frac{1}{k^{2}} \int_{r}^{\infty} \frac{\sin (k r-l \pi / 2)}{r^{2}} d r
$$

where $c_{l}$ is the value of the integral (10) from 0 to $\infty[16]$,

$$
c_{l}=\frac{\sqrt{\pi} \Gamma(l / 2)}{2(l+1) \Gamma(l / 2+1 / 2)} .
$$

Thus, for sufficiently large values of $r$, the operator $F_{l}$ reaches a constant value. This is in contrast to the LWA where the values of the length operator increase indefinitely.

Comparison between the full operator and its LWA is simplified for $l_{i}=0$ and $l_{f}=1$ when the only value allowed to $l$ is unity. In Fig. 1 we plot the function $3 F_{1}(r)$ together with $3 F_{1}(r)=r$, the latter representing the LWA. Then, $c_{1}$ $=\pi / 4$. Putting $r_{0}=3 \pi / 4 k$, Fig. 1 suggests the following model of practical value:

$$
3 F_{1}(r)=\left\{\begin{array}{cc}
r, & r \leqslant r_{0} \\
r_{0}, & r \geqslant r_{0}
\end{array}\right.
$$

In words, the LWA is followed up to $r_{0}$ while a constant value is assumed for larger values of $r$. This model can serve as a rough test for the region of validity of the LWA. The smaller the value of $k$ is, the larger is the region of validity. Hence, if the radial part of either the initial or the final wave function falls off exponentially, for $r \geqslant r_{0}$, then the LWA is valid. It becomes obvious that for transitions between high Rydberg or between scattering states, the LWA is not a satisfactory approximation.

\section{B. Paramagnetic field operator}

This is the second term of the Hamiltonian (5). In the general expression (7), where now $l_{\min }=0$ and $X(t)$ $=B_{0}(t) e^{-i \omega t}$, we put

$$
F_{l}(r)=\frac{1}{r^{2}} \int_{0}^{r} r^{\prime} j_{l}\left(k r^{\prime}\right) d r
$$

and

$$
\begin{aligned}
\Theta_{l}(\theta, \varphi)= & \frac{1}{2 i} \sqrt{4 \pi /(2 l+1)} \\
& \times\left[\sqrt{l(l+1)}\left(Y_{l}^{1}-Y_{l}^{-1}\right)+2 Y_{l}^{0}\left(\hat{l}_{+}-\hat{l}_{-}\right)\right],
\end{aligned}
$$

where

$$
\hat{l}_{ \pm} Y_{l}^{m}=\sqrt{(l \mp m)(l \pm m+1)} Y_{l}^{m \pm 1} .
$$

In this case, the allowed transitions have $\Delta m= \pm 1$.

\section{Diamagnetic field operator}

This is a more complicated operator. We first split it into two parts, each one corresponding to a different angular part, obtained by using the formula

$$
(\hat{r} \times \hat{y})^{2}=\frac{1}{2}\left(1+\cos ^{2} \theta\right)+\frac{1}{2} \sin ^{2} \theta \cos 2 \phi .
$$

We now reduce the double integration, which is implicit in the third term of Eq. (5), to a single one, using the fact that the integrand depends only on the sum of the two variables. It follows that

$$
\begin{aligned}
& \int_{0}^{1} \lambda^{\prime} d \lambda^{\prime} \int_{0}^{1} \lambda^{\prime \prime} d \lambda^{\prime \prime} \exp \left[i\left(\lambda^{\prime}+\lambda^{\prime \prime}\right) \vec{k} \cdot \vec{r}\right] \\
& =\frac{1}{3} \int_{0}^{1}\left(\lambda^{3}-3 \lambda+2\right) \exp (i \lambda \vec{k} \cdot \vec{r}) d \lambda-\frac{4}{3} \\
& \quad \times \int_{0}^{1}\left(2 \lambda^{3}-3 \lambda+1\right) \exp (i 2 \lambda \vec{k} \cdot \vec{r}) d \lambda .
\end{aligned}
$$


The first part of the angular operator (16) allows transitions with $\Delta m=0$ [now $\left.l_{\min }=0, X(t)=B_{0}{ }^{2}(t) e^{-i 2 \omega t}\right]$. The radial factor corresponding to this part is, following Eq. (17),

$$
\begin{aligned}
F_{l}(r)= & \frac{1}{6 r^{2}} \int_{0}^{r} r^{\prime 3} f_{l}\left(k r^{\prime}\right) d r^{\prime}-\frac{1}{2} \int_{0}^{r} r^{\prime} f_{l}\left(k r^{\prime}\right) d r^{\prime} \\
& +\frac{1}{3} r \int_{0}^{r} f_{l}\left(k r^{\prime}\right) d r^{\prime}-\frac{4}{3 r^{2}} \int_{0}^{r} r^{\prime 3} f_{l}\left(2 k r^{\prime}\right) d r^{\prime} \\
& +2 \int_{0}^{r} r^{\prime} f_{l}\left(2 k r^{\prime}\right) d r^{\prime}-\frac{2}{3} r \int_{0}^{r} r^{\prime 3} f_{l}\left(2 k r^{\prime}\right) d r^{\prime},
\end{aligned}
$$

where

$$
f_{l}(k r)=\left(1+\frac{l(l+1)}{2 k^{2} r^{2}}\right) j_{l}(k r)+\frac{1}{k r} j_{l}^{\prime}(k r) .
$$

$f_{l}(k r)$ is obtained by using Eq. (A6) and eliminating the resulting second derivative of the Bessel function using the Bessel differential equation. Then, the angular factors are

$$
\Theta_{l}(\theta, \phi)=\sqrt{l(l+1) / 4 \pi} Y_{l}^{0}(\theta, \phi) .
$$

Although Eq. (18) looks complicated, the numerical evaluation of $F_{l}(r)$ is straightforward, the Bessel function and its derivative being simultaneously produced by the same routine [17].

We now turn to the second part, for which the allowed transitions have $\Delta m= \pm 2$. Application of Eq. (A5) gives $\left[l_{\min }=2, X(t)=B_{0}^{2}(t) e^{-i 2 \omega t}\right]$,

$$
\begin{aligned}
F_{l}(r)= & \frac{1}{24 k^{2} r^{2}} \int_{0}^{r} r^{\prime} j_{l}\left(k r^{\prime}\right) d r^{\prime}-\frac{1}{8 k^{2}} \int_{0}^{r} \frac{1}{r^{\prime}} j_{l}\left(k r^{\prime}\right) d r^{\prime} \\
& +\frac{r}{12 k^{2}} \int_{0}^{r} \frac{1}{r^{\prime 2}} j_{l}\left(k r^{\prime}\right) d r^{\prime}-\frac{1}{3 k^{2} r^{2}} \int_{0}^{r} r^{\prime} j_{l}\left(k r^{\prime}\right) d r^{\prime} \\
& +\frac{1}{2 k^{2}} \int_{0}^{r} \frac{1}{r^{\prime}} j_{l}\left(k r^{\prime}\right) d r^{\prime}-\frac{r}{8 k^{2}} \int_{0}^{r} \frac{1}{r^{\prime 2}} j_{l}\left(k r^{\prime}\right) d r^{\prime}
\end{aligned}
$$

and

$$
\begin{aligned}
\Theta_{l}(\theta, \phi)= & \sqrt{[4 \pi /(2 l+1)] l\left(l^{2}-1\right)\left(l^{2}-4\right)} \\
& \times\left(Y_{l}^{2}+Y_{l}^{-2}\right) .
\end{aligned}
$$

\section{APPLICATIONS: COMPARISON OF THE EDA WITH THE EXACT TREATMENT}

We concentrate on the electric field, whose effects are much larger than those of the magnetic one. [See Eq. (5), where the paramagnetic operator is divided by $c$ and the diamagnetic one by $c^{2}$.]

As an application, we calculated the radial matrix elements corresponding to the electric-dipole-allowed transition $n s \rightarrow n p$ and to the dipole-forbidden transition $n s \rightarrow n d$ of

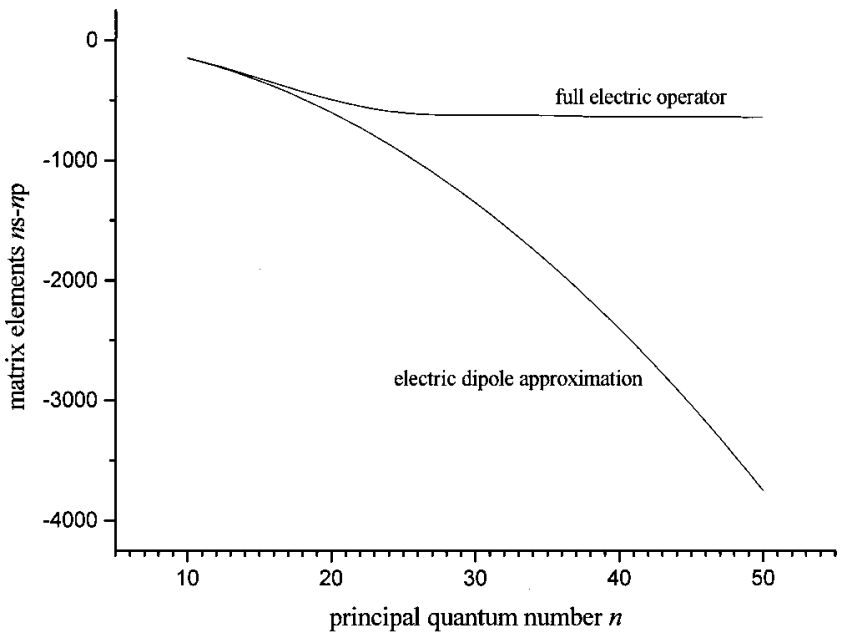

FIG. 2. Plot of the transition matrix element (radial part) $n s$ $\rightarrow n p$ calculated with the full electric operator, versus the EDA result $-(3 / 2) n \sqrt{n^{2}-1}$.

hydrogen, for values of $n$ up to 50 and for $\omega=0.5$ a.u. (the hydrogenic ionization potential). Note that $k=\omega / c$, where $c=137.037$ in atomic units. In the first case, $r_{0}$ is equal to 645.77 a.u. In Fig. 2 we plot the radial matrix elements $\left\langle u_{n 0}\left|3 F_{1}(r)\right| u_{n 1}\right\rangle$ and $\left\langle u_{n 0}|r| u_{n 1}\right\rangle$. The latter is equal to $-\frac{3}{2} n \sqrt{n^{2}-1}$ [18]. For values of $n$ up to 10 , the values of the two matrix elements are essentially identical. However, as $n$ increases beyond 10, a discrepancy starts, the former matrix element approaching a value equal to $r_{0}$. Noting that for sufficiently large values of $n$ the hydrogenic functions concentrate approximately at a distance $2 n^{2}$, with the convention of having positive values close to the origin, and that the overlap $\left\langle u_{n 0} \mid u_{n 1}\right\rangle \rightarrow-1$, the behavior of the matrix element can be explained by the simple model Eq. (12) described in Sec. III.

In the case of the dipole-forbidden transition, in which case $\left\langle u_{n 0} \mid u_{n 2}\right\rangle \rightarrow 1$, a similar behavior of the corresponding matrix element is observed; see Fig. 3.

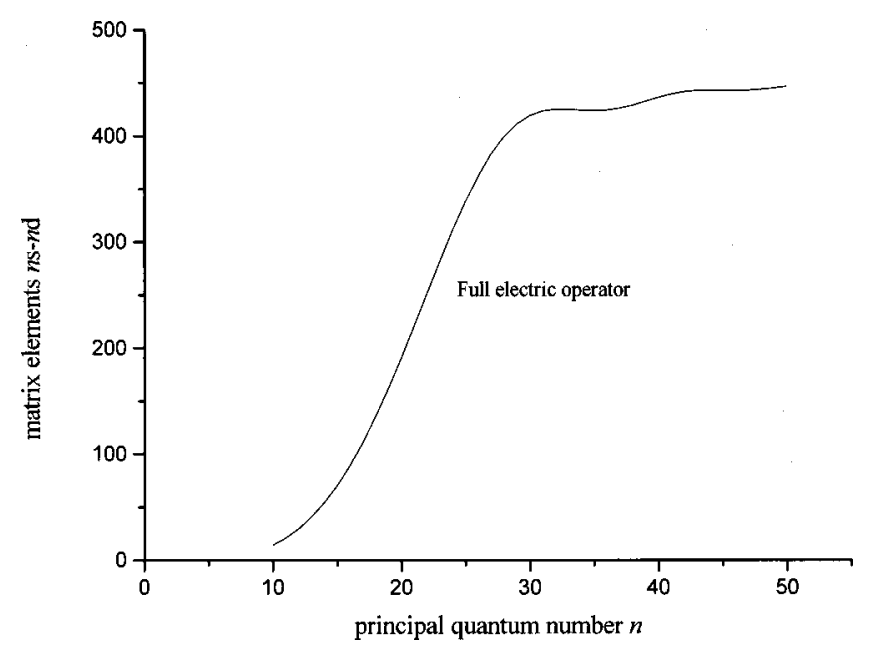

FIG. 3. Plot of the transition matrix element (radial part) $n s$ $\rightarrow n d$ calculated with the full electric operator (forbidden electricdipole transition). 


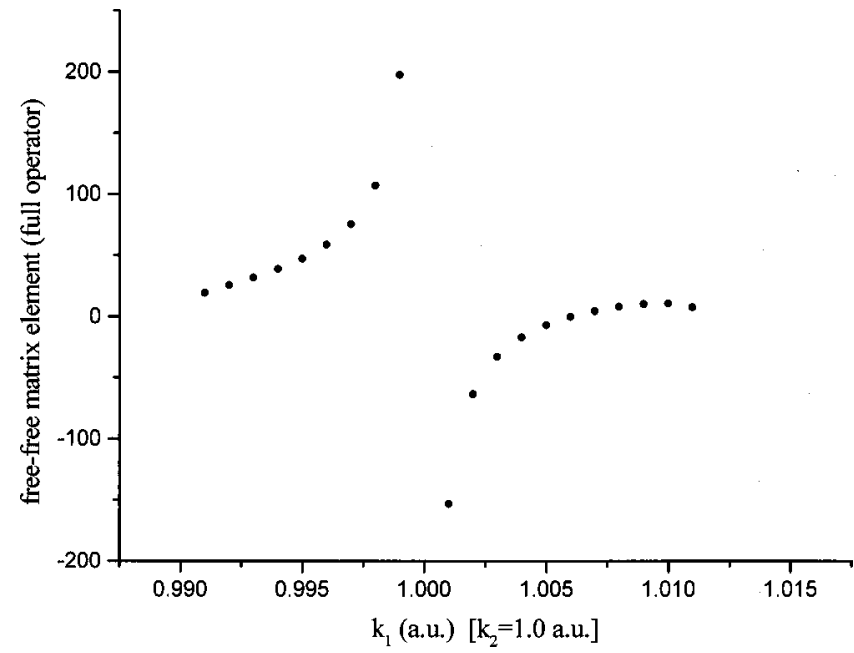

FIG. 4. Plot of the transition matrix element (radial part) $k_{1} s$ $\rightarrow k_{2} p$ between energy-normalized Coulomb wave functions calculated with the full electric operator.

The hydrogenic wave functions of the above example as well as the wave functions of the continuous spectrum used in the following calculation are computed via numerical solution of the differential equation that they satisfy. For highly excited states, this is the fastest and most accurate method and is immediately applicable to the one-electron states of atoms other than hydrogen.

\section{Free-free transitions for the Coulomb potential}

The matrix elements between functions of the continuous spectrum can be evaluated as follows. We first choose a conveniently large value $b$ such that $j_{1}(b)$, appearing in the electric-field operator, is obtained within a given accuracy by the asymptotic series of the spherical Bessel function. In the region $k r<b$, the expression (8) is used and $F_{1}$ is calculated numerically. In the outer region, $k r>b$, the expression (10) is used and an overlap appears due to the constant term. The overlap between Coulomb functions of different angular momenta provides the usual $\delta$ function singularity plus a principal-value kernel. The calculation is analogous to that showing the $\delta$ orthonormality between Coulomb functions of the same angular momentum, e.g., [19]. For atoms other than hydrogen, the phase shift of the asymptotic part of the wave functions has to be taken into account.

A plot of the matrix element is given in Fig. 4, while the corresponding plot for the dipole operator is given in Fig. 5. In the latter case, the integral has been expressed by Gordon in terms of hypergeometric functions [20]. Comparing the two figures, we can see that the matrix elements not only differ dramatically in magnitude, but also differ qualitatively in their behavior about the singular point $k_{1}=k_{2}$, since the EDA results in a $\delta^{\prime}$ singularity and a second-order principalvalue kernel [21].

\section{CONCLUSION}

By implementing the theory of the interaction of the electromagnetic field with an atomic state in terms of the multi-

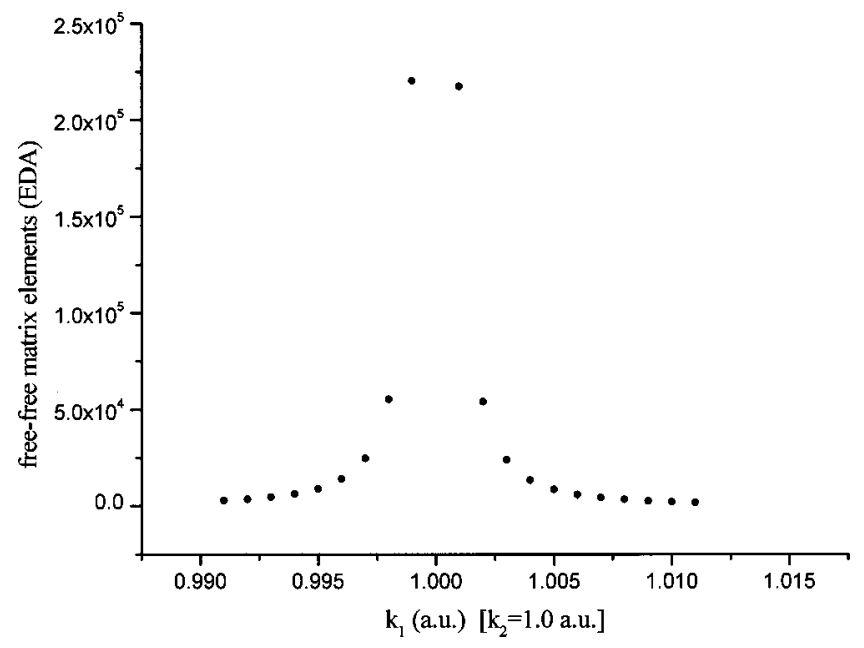

FIG. 5. Plot of the transition matrix element (radial part) $k_{1} s$ $\rightarrow k_{2} p$ between energy-normalized Coulomb wave functions calculated in the EDA (Gordon formula).

polar Hamiltonian, Eq. (5), we developed and applied formulas for the practical calculation of the exact matrix elements of the electric, paramagnetic, and diamagnetic interactions, using numerical (and, therefore, general) one-electron wave functions. The prototypical examples that we used showed that the widely employed EDA is not justified for the calculation of matrix elements between highly excited RydbergRydberg, Rydberg-scattering and scattering-scattering states. Model (12) quantifies this statement in a transparent way. The error is especially large for transitions between states of the same energy, which play a crucial role in the formation of wave packets excited from a lower state via laser pulses (e.g., [5-9], EDA calculations).

The results of this work are being implemented in computationally demanding investigations of the excitation and evolution of highly excited Rydberg levels. Preliminary calculations [22] show that when hydrogen is excited to the $n$th shell from its ground state with laser pulses of frequencies $\omega=0.5\left(1-1 / n^{2}\right)$, the results for the time-dependent population of initial and final levels from the use of the full interaction (with the on-shell couplings taken into account) start differing from those obtained from the EDA when $n$ becomes larger than 10 . Already for $n=25$ the differences are very significant [22] and force us to suggest that it is doubtful whether the theory and understanding of Rydberg wavepacket formation and dynamics is reliable within the framework of the EDA.

\section{APPENDIX}

The single-mode vector potential is written as

$$
\vec{A}=A_{0}(t) \vec{x} e^{i \vec{k} \cdot \vec{r}-i \omega t}+\text { c.c. }
$$

The corresponding electric field $\left[\vec{E}_{T}=-(1 / c)(\partial / \partial t) \vec{A}\right]$ is written as

$$
\vec{E}_{T}=E_{0}(t) \hat{x} e^{i \vec{k} \cdot \vec{r}-i \omega t}+\text { c.c. }
$$


Also, since $\vec{B}=\vec{\nabla} \times \vec{A}=i \vec{k} \times \vec{A}$,

$$
\vec{B}=i k A_{0}(t) \hat{y} e^{i \vec{k} \cdot \vec{r}-i \omega t}+\text { c.c. }
$$

The expansion of the plane wave in spherical waves is

$$
e^{i \vec{k} \cdot \vec{r}}=e^{i k r \cos \theta}=\sum_{l=0}^{\infty} i^{l}(2 l+1) j_{l}(k r) P_{l}(\cos \theta) .
$$

As we proved in [4],

$$
\sin ^{m} \theta e^{i k r \cos \theta}=\left(\frac{i}{k r}\right)^{m} \sum_{l=m}^{\infty} i^{l}(2 l+1) j_{l}(k r) P_{l}^{m}(\cos \theta)
$$

while differentiating Eq. (A4) $m$ times with respect to $k r$ gives

$$
\cos ^{m} \theta e^{i k r \cos \theta}=(i)^{m} \sum_{l=0}^{\infty} i^{l}(2 l+1) j_{l}^{(m)}(k r) P_{l}(\cos \theta) .
$$

[1] D. M. Brink and G. R. Satchler, Angular Momentum, 3rd ed. (Clarendon, Oxford, 1993).

[2] R. Loudon, The Quantum Theory of Light, 2nd ed. (Clarendon, Oxford, 1983).

[3] D. P. Craig and T. Thirunamachandran, Molecular Quantum Mechanics, (Dover, Mineola, NY, 1998).

[4] Th. Mercouris, Y. Komninos, S. Dionissopoulou, and C. A. Nicolaides, J. Phys. B 30, 2133 (1997).

[5] J. D. Corless and C. R. Stroud, Jr., Phys. Rev. Lett. 79, 637 (1997).

[6] H. M. Nilsen and J. P. Hansen, Phys. Rev. A 63, 011405(R) (2001)

[7] H. G. Muller and L. D. Noordam, Phys. Rev. Lett. 82, 5024 (1999); L. B. Madsen, ibid. 85, 898 (2000); H. G. Muller and L. D. Noordam, ibid. 85, 899 (2000).

[8] P. Parzynski, M. Sobczak, and A. Wojcik, Phys. Rev. A 63, 063413 (2001); J. Phys. B 34, 3629 (2001).

[9] B. S. Mecking and P. Lambropoulos, Phys. Rev. Lett. 83, 637 (1997)

[10] L. B. Madsen, J. P. Hansen, and H. M. Nilsen, Comput. Phys. Commun. 120, 231 (1999).

[11] W. E. Lamb, Jr., R. R. Schlicher, and M. O. Scully, Phys. Rev.
A 36, 2763 (1987).

[12] E. A. Power and S. Zienau, Philos. Trans. R. Soc. London, Ser. A 251, 427 (1959).

[13] E. A. Power and T. Thirunamachandran, Proc. R. Soc. London, Ser. A 372, 265 (1980).

[14] R. G. Wooley, Adv. Chem. Phys. 33, 153 (1975).

[15] Y. Aharonov and K. Au, Phys. Lett. 95A, 412 (1983); D. H. Kobe and K.-H. Yang, Phys. Rev. A 32, 952 (1985).

[16] I. S. Gradshteyn and I. M. Ryzhik, Table of Integrals, Series and Products, 6th ed., edited by A. Jeffrey (Academic, San Diego, 2000).

[17] W. H. Press, S. A. Teukolsky, W. T. Vettering, and B. P. Flannery, Numerical Recipes, 2nd ed. (Cambridge University Press, Cambridge, England, 1992).

[18] H. A. Bethe and E. E. Salpeter, Quantum Mechanics of One and Two-Electron Atoms (Plenum, New York, 1977).

[19] M. J. Seaton, Rep. Prog. Phys. 46, 167 (1983).

[20] A. Maquet, Phys. Rev. A 15, 1101 (1977).

[21] V. Veniard and B. Piraux, Phys. Rev. A 41, 4019 (1990).

[22] Th. Mercouris, Y. Komninos, and C. A. Nicolaides, J. Phys. B 35, 1439 (2002). 